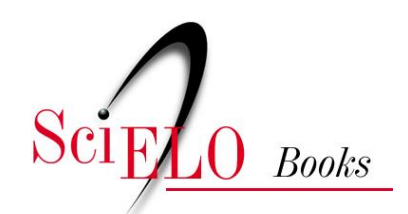

\title{
Persona, sentido de la vida, trascendencia ante la increencia e indiferencia religiosa
}

\author{
Roberto Carlos Cuenca Jiménez
}

\section{SciELO Books / SciELO Livros / SciELO Libros}

CUENCA JIMÉNEZ, R.C. Persona, sentido de la vida, trascendencia ante la increencia e indiferencia religiosa. In: IZA VILLACÍS, V.A., ed. Persona, educación y filosofía: reflexiones desde la educación universitaria [online]. Quito: Editorial Abya-Yala, 2018, pp. 123-141. ISBN: 978-9978-10493-4. https://doi.org/10.7476/9789978104934.0008.

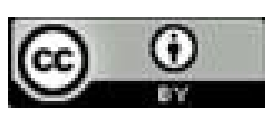

All the contents of this work, except where otherwise noted, is licensed under a Creative Commons Attribution 4.0 $\underline{\text { International license. }}$

Todo o conteúdo deste trabalho, exceto quando houver ressalva, é publicado sob a licença Creative Commons Atribição 4.0.

Todo el contenido de esta obra, excepto donde se indique lo contrario, está bajo licencia de la licencia $\underline{\text { Creative }}$ Commons Reconocimento 4.0. 


\title{
Persona, sentido de la vida, trascendencia ante la increencia $e$ indiferencia religiosa
}

\author{
Roberto Carlos Cuenca Jiménez, Msc ${ }^{1}$
}

\section{Resumen}

El presente trabajo contextualiza la realidad de la persona en su integridad y sentido de la trascendencia en la vida de las personas y un análisis de la propia realidad cultural respecto de la increencia e indiferencia desde el fenómeno religioso que parte desde una fundamentación teórica y comparación, la misma que se contextualiza con aportes del magisterio, planteamientos de teólogos y la incidencia del pensamiento filosófico que se unen a explicar la realidad cultural y social de las propias realidades humanas, desde unos principios fundamentales de las manifestaciones religiosas y educativas, escenarios donde el ser humano interactúa con su realidad personal.

1 Doctorando en el Programa de Doctorado Interuniversitario en Comunicación conjunto de las Universidades de Sevilla, Málaga, Huelva y Cádiz. Máster en Evaluación, Gestión y Dirección de Calidad Educativa (Universidad de Sevilla). Máster en Ciencias de la Familia (Asesoramiento, Orientación, Mediación e Intervención Familiar) en la Universidad Santiago de Compostela. Diplomado en Gestión del Talento Humano en la UTPL, Licenciatura Ciencias de la Educación, mención Ciencias Humanas y Religiosas (UTPL). Filosofía y Teología Universidad de Navarra-España. Docente investigador Sección de Filosofía y Teología. Universidad Técnica Particular de Loja (Ecuador). rccuenca@utpl.edu.ec 
En el caso metodológico se aplicó encuestas y se utilizó los métodos deductivo, inductivo, sintético y descriptivo. En el contexto general se evidencia la incidencia de principios, valores, la cultura, lo social, en las manifestaciones hacia lo trascendente.

Palabras clave: Persona, sentido, educación, religión, increencia, sociedad, trascendencia.

\section{Abstract}

The present work contextualizes the reality of the person in its integrity and sense of the transcendence in the life of the people and an analysis of the cultural reality itself regarding the unbelief and indifference from the religious phenomenon that starts from a theoretical foundation and comparison, the same one that is contextualized with contributions of the magisterium, theologians' expositions and the incidence of the philosophical thought that are united to explain the cultural and social reality of the own human realities, from fundamental principles of the religious and educational manifestations, scenarios where the being human interacts with your personal reality.

In the methodological case, surveys were applied and deductive, inductive, synthetic and descriptive methods were used. In the general context the incidence of principles, values, culture, the social, in the manifestations towards the transcendent is evident.

Keywords: Person, sense, education, religion, unbelief, society, transcendence.

\footnotetext{
"El hombre, viviendo en fidelidad al único Dios, se experimenta a sí mismo como quien es amando por Dios y descubre la alegría en la verdad y en la justicia; la alegría en Dios que se convierte en su felicidad esencial" (Ratzinger, Deus Caritas).
}

\section{Introducción}

El término persona se deriva del verbo latino personare que significa resonar; y de la versión del griego prosopón que significa másca- 
ra. La palabra dignidad proviene del latín, de la raíz de dignitas = calidad de digno y del verbo decet $=$ justo, honesto, de donde derivan los sustantivos decor y decís, es decir, "Algo que tiene excelencia y dignidad en virtud de su belleza y decoro".

En el texto de Antropología De Angelis y Zordan (2006) se menciona que "persona" es un término que quiere designar al hombre en su totalidad e indicar al mismo tiempo el carácter esencial, lo que diferencia del resto de la naturaleza. Pero "la noción de persona adquiere su significado más pleno, a partir del dogma de la Trinidad, en los primeros siglos del cristianismo, a partir del término latino persona, correspondiente con los términos griegos prosopon e hypostasis, fueron elaborados por los padres de la Iglesia a la luz de la revelación cristiana”.

La Real Academia de la Lengua define a la dignidad como la "gravedad y decoro de las personas en la manera de comportarse". El ser humano es digno porque trasciende toda temática biológica: Ser dotado de inteligencia y libertad. Al herir la dignidad de cualquier persona se está hiriendo a sí mismo, porque los demás nunca le son ajenos. La dignidad de la persona no es consecuencia de su obrar moralmente, sino que, ésta, le es intrínseca al hombre por el hecho mismo de ser persona.

Es por eso, que la vida personal es una constante lucha frente a las dificultades, problemas y circunstancias que se presentan a lo largo de nuestra existencia. "En una sociedad centrada en lo inmediato no es posible forjar el carácter del ser humano, disminuyendo su importancia como persona y siendo fácilmente manipulable", lo cual no genera cambios sustanciales que nos ayude a repensar nuestras ideas de verdad, dignidad, libertad, para cimentarlas en el terreno fértil, más que un ámbito superficial que dominan nuestros propios egoísmos.

En este mismo contexto de felicidad debe tener presente la identificación propia del ser humano que debe estar fundada en el amor, que constituye el horizonte vital de la educación y formación de la persona; que al respecto S.S. Juan Pablo II, en Redemptor Ho- 
minis, $\mathrm{n}^{\circ} 10$, explicaba en su reflexión antropológica: “(...) el hombre no puede vivir sin amor... (el hombre) permanece para sí mismo un ser incomprensible, su vida se vacía de sentido, si no se revela el amor, si no se encuentra el amor, si no lo experimenta y hace propio, si no participa en él vivamente".

Sería importante la reflexión sobre ciertos acontecimientos que nos causan sufrimiento, desesperación; quizás pueda darse una razón de estos hechos. Sin embargo, los grandes interrogantes existenciales y personales: ¿Cuál es el sentido último de nuestra existencia? ¿Por qué y para qué vivimos? ¿Qué busco yo con mi vida, con toda mi vida? ¿Tiene sentido la vida y en qué medida? Es fundamental aclarar que las respuestas que demos a estos planteamientos desde nuestra vivencia, debe iluminar la problemática concreta y existencial primero de cada uno, porque "nadie da de lo que no tiene sino de lo que abunda en lo profundo del corazón”, de nuestro ser.

Por ello, el ser humano, al interrogarse por su propia existencia, su origen, desarrollo, finalidad y su proyección trascendente, siempre se encuentra en permanente búsqueda de superación y perfección como persona. Es fundamental aclarar que las respuestas que demos a estos planteamientos desde nuestra vivencia, debe iluminar la problemática concreta y existencial primero de cada uno, porque "nadie da de lo que no tiene sino de lo que abunda en lo profundo del corazón”, de nuestro ser.

El llegar a conocerse a uno mismo, el enfrentarse ante la realidad tal como es, en algunas veces es costoso, difícil, pero nunca imposible de superar todos los obstáculos que se presenten en la vida, cuando los mismos son vivenciados y apoyados en la caridad perfecta del amor, a lo cual el Apóstol San Pablo en la 1ra Carta a los Corintios 13 describía la vivencia del amor; al mismo tiempo Juan Pablo II nos recordaba que "el trabajo más importante no es el de la transformación del mundo, sino el de la transformación de nosotros mismos; por lo que la paz exige cuatro condiciones esenciales: verdad, justicia, amor y libertad". 
Reflexionar, dar sentido y respuesta a nuestra vida respecto de la diferencia e increencia, en el contexto del fenómeno religioso, es primordial asumir un compromiso personal, pero también debemos cuestionarnos ¿Cómo desarrollar el espíritu crítico reflexivo de cada uno frente a realidad actual? En tal virtud hay que valorar, exponer nuestras propias situaciones y actitudes positivas como personas que nos lleve a dar respuesta y sentido a nuestra vida, en el ámbito profesional y en relación con los demás.

\section{Contextos}

\section{Increencia e indiferencia religiosa}

Referirnos a la increencia e indiferencia es necesario, iniciar, diferenciando los términos para que no cause confusión. Al respecto, el Consejo Pontificio para la Cultura (2004) acentúa la incidencia de la cultura:

Más que de convencer, la evangelización de la cultura trata de preparar un terreno favorable a la escucha, es una especie de pre evangelización. Si el problema fundamental es la indiferencia, el primer deber al que la Iglesia no puede renunciar es el de despertar la atención y suscitar el interés de las personas.

Es decir, señala la importancia de identificar algunos puntos de anclaje para el anuncio del Evangelio, proposiciones que ofrecen diferentes orientaciones aplicados en una pastoral de la cultura, con el fin de ayudar a la Iglesia a proponer la fe cristiana respondiendo al desafío de la increencia e indiferencia religiosa al alba del nuevo milenio.

Según el diccionario de Filosofía, comprender el significado de creer, creencia es dar por cierta una cosa que no se ha visto o demostrado. Se puede referir a hechos naturales o religiosos. Es un modo imperfecto de conocer, pero resulta necesario dadas las limitaciones del conocimiento humano, es decir, si solo conociéramos lo que hemos visto o demostrado, nuestro conocimiento de la realidad sería notoriamente pobre y nos dejaría indefensos ante la vida. 
En el ámbito del fenómeno religioso es relevante el aporte de Sánchez (2008 citado en Tamayo, 2004) en su análisis sobre la pluralidad como principio, anotaciones para una Teología Fundamental pluralista, señala:

El fenómeno religioso, de por sí complejo en todo tiempo y lugar, reviste hoy una complejidad especial, debido, en buena medida, a la influencia que vienen ejerciendo en él las profundas mutaciones socio-culturales producidas en nuestro mundo. De ahí la dificultad de hacer un análisis matizado y un diagnóstico preciso de la situación socio-religiosa y cultural actual. Ello obliga a adoptar una actitud de prudencia para evitar toda generalización infundada, sugerir más que afirmar con rotundidad, insinuar más que hacer declaraciones contundentes, ofrecer pistas más que establecer seguridades y exponer dudas más que proclamar certezas (p. 104).

En este sentido, los últimos datos proporcionados por el Instituto Nacional de Estadísticas y Censos (INEC) relativos a la filiación religiosa en el Ecuador, muestran el profundo sentimiento religioso existente entre los habitantes del país: el 91,95\% de las personas encuestadas afirman tener una religión, frente al 7,94 \% de personas que se auto identifican como agnósticas y el 0,11\% que declaran ser ateas (INEC, 2012). Entre las religiones recogidas en la encuesta, las opciones de respuesta incluyen la religión cristiana católica, cristiana evangélica, Testigos de Jehová, mormones, budistas, religiones indígenas y afroamericanas, judaísmo, hinduismo y religión islámica, entre otras. Pese a la diversidad religiosa que se muestra, se destaca de forma significativa que el $80,44 \%$ de las personas encuestadas afirman profesar la religión cristiana católica (INEC, 2012).

El dato anterior se vincula directamente con los factores que integran la formación educativa a través de la institución eclesial. El fenómeno religioso, entendido como expresión de identidad individual y colectiva, supone una manifestación viva de los modos en los que se articula la realidad nacional del país. Por ello, las propuestas encaminadas a la interpretación e integración del fenómeno religioso 
en los modelos educativos deberán situar el análisis del objeto de estudio desde contextos específicos que respondan a la realidad señalada.

En este mismo ámbito, según Vitoria (2012) en su texto Vientos de cambio, capítulo II relacionado con los cuatro signos actuales del reinado de Dios, uno de estos signos está el pluralismo religioso donde resalta:

Una de las muchas paradojas que caracteriza a nuestro mundo es que la creciente globalización va acompañada de nuevas diferenciaciones culturales y religiosas. Cosmopolitismo y particularidad no son realidades opuestas, sino complementarias. Nos encontramos en una época de diferencias entrelazadas. Las diversas culturas presentes en nuestro mundo no solamente coexisten unas junto a otras, sino que además mantienen entre sí relaciones dinámicas que, como nos recuerda un informe de la UNESCO, necesitamos y debemos aprender a orientar no hacia una confrontación, sino a una coexistencia fecunda y una armonía intercultural (p. 23)

En el contexto de la indiferencia religiosa Girardi (1967) citado de la Gaudium et Spes:

Se designa ordinariamente en la actualidad una forma peculiar de increencia. Una actitud vital en la que el sujeto no acepta ni rechaza a Dios, sino que prescinde de él, organizando su vida totalmente al margen de la práctica religiosa, Se trata; pues, de una actitud que se caracteriza por el "desinterés y la desafección hacia Dios y la dimensión religiosa de la existencia", actitud a la que se refería el concilio Vaticano II al mencionar, entre las formas de ateísmo, el ateísmo de aquellos que "ni siquiera plantean la cuestión de la existencia de Dios porque, al parecer, no sienten inquietud religiosa alguna y no perciben el motivo de preocuparse por el hecho religioso" (GS 19,2).

A modo de aclaración, el autor antes mencionado reitera la indiferencia religiosa es la falta de interés por Dios y por la religión. Dios, exista o no exista, no es un valor. En este sentido, se trata de una insensibilidad a la misma cuestión religiosa, es una falta de preocupación "en materia religiosa". Estos hechos son consecuencias fruto de una constante insensibilidad en materia religiosa, la falta de 
cuestionamientos de la persona sobre el sentido de lo humano, de su destino y del mundo.

Además, en este mismo sentido, para González de Cardedal (1985) respecto al increencia religioso se resalta como "aquella forma de vida en la que Dios no está presente como luz que alumbra la existencia, no da razón del origen de la realidad y del sentido de la historia, no funda el hecho mismo de existir ni la vida personal del hombre".

De esta manera, podemos comprender, a nuestro entender, dentro de la más amplia filosofía moral, es decir, desde el ámbito de las actitudes y comportamientos de la persona, conforme lo indica Llano (2005), en su libro La vida lograda. ¿Quién no quiere mirar a su vida y poder decir algo semejante de ella? Todos aspiramos a vivir una vida completa, entera, lograda. Eso está muy claro. Y también lo está que no todos lo conseguimos. De hecho, hay personas felices y personas desdichadas. Algunas logran su vida y otras la malogran, la pierden, fracasan en ella. No fracasan en este o en aquel aspecto, sino que fracasan en sí mismas, precisamente como personas.

Sin embargo, en este mismo planteamiento, según Izquierdo (2006) el término "increencia" es un fenómeno complejo y difícil de fijar, que tiene como rasgo común la tendencia a dejar de lado las creencias religiosas. Se puede considerar increencia la actitud de prescindir de Dios o de cualquier realidad trascendente bien como resultado de una reflexión que conduce a justificar la no existencia de Dios o la imposibilidad de conocerle o bien simplemente porque no se presta interés a esta cuestión.

\section{Anuncio del kerigma}

En el proceso formativo y profesional como personas es importante asumir una reflexión del accionar humano, en el sentido de saber discernir en nuestras vidas los comportamientos que se identifican en la propia experiencia humana, en la constante búsqueda de una sociedad más justa y digna. Iniciamos reflexionando el significado de Ke- 
rigma según Catequesi Tradendae hace énfasis "al primer anuncio del evangelio o predicación misional por medio del kerigma para suscitar la fe" (CT18). Además, especifica que "el kerigma evangélico — primer anuncio lleno de ardor que un día transformó al hombre y lo llevó a la decisión de entregarse a Jesucristo por la fe” (CT25).

Es fundamental profundizar, reflexionar y comprender en nuestra vida lo que hemos dejado de hacer, lo que hemos hecho mal, para que a la luz de la revelación en la persona de Cristo nos fortalezca nuestra vida para ser mejores personas integralmente. El mismo apóstol san Pablo exhortaba: "Todo cuanto hay de verdadero, de noble, de justo, de puro, de amable, de honorable, todo cuanto sea virtud y cosa digna de elogio, todo eso tenedlo en cuenta" (Flp 4,8). La persona virtuosa está en el camino de ser sabio, porque sabe cómo hacer realidad sus sueños y el de los demás.

En esta dirección y sentido es dar respuesta a nuestro accionar desde el evangelio, y la misma Iglesia resalta de que todos los cristianos, hoy, puedan "evangelizar a partir del centro de la vida y hasta las raíces de la vida” (Pablo VI, en la Evangelii Nuntiandi n. 19.). Así mismo la Redemptoris Missio considera que el anuncio tiene la prioridad permanente en la misión: La Iglesia no puede substraerse al mandato explícito de Cristo; no puede privar a los hombres de la "Buena Nueva" de que son amados y salvados por Dios (RM,44).

En este sentido, es de trascendental valor, promover todos aquellos aspectos que aumenten la conciencia de pertenencia a la Iglesia, a una mejor participación en el culto y los sacramentos y a una más amplia inserción en la labor evangelizadora de la Iglesia; pero teniendo en cuenta el mundo de las expresiones populares y el alma del pueblo, incluso en la religiosidad se pueden distinguir elementos culturales, antropológicos y sociales que iluminan y dan sentido a la pastoral, a la liturgia y a la vida de cada creyente, indiferente religioso.

El anuncio del evangelio, de la Buena Nueva de Jesucristo, al respecto la Chrfistifidelis Laici indica: "la Iglesia tiene que dar hoy un 
gran paso adelante en su evangelización; debe entrar en una nueva etapa histórica de su dinamismo misionero". Pero el problema misionero se presenta actualmente a la Iglesia con una amplitud y con una gravedad tales, que sólo una solidaria asunción de responsabilidades por parte de todos los miembros de la Iglesia - tanto personal como comunitariamente - puede hacer esperar una respuesta eficaz (CL, 35).

Precisamente la experiencia del encuentro ontológico con el Absoluto, debe ser el punto de partida, el núcleo de la fe existente y el proceso de maduración cristiana para profundizar, sentir, pensar y actuar a la altura de Cristo; como dice San Pablo: "ninguno de vosotros vive para sí mismo; como tampoco muere nadie para sí mismo. Si vivimos, vivimos para el Señor; y si morimos, morimos para el Señor. Así que, ya vivamos ya muramos somos del Señor" (Rm 14,7-8). Es decir, reconocer lo que es Cristo en nuestra vida afecta entonces por entero a la conciencia con la que vivimos, como lo indica el mismo Cristo: "Yo soy el Camino, la Verdad y la Vida" (Jn 14,6). Surge entonces, la necesidad de crear un ambiente de reflexión y concientización en la vida de cada cristiano, con el propósito de analizar aquellas actitudes y comportamientos que no están en relación con la fe que profesamos. La Palabra que ilumina las entrañas del ser humano y da sentido el encuentro con Cristo que se manifiesta en la propia experiencia humana que surge de la vivencia en comunidad.

En este sentido según la Redemptoris Missio, está claro, que Jesús vino a traer la salvación integral, que abarca al hombre entero y a todos los hombres, abriéndoles a los admirables horizontes de la filiación divina. (RM11). El anuncio tiene por objeto a Cristo crucificado, muerto y resucitado: en él se realiza la plena y auténtica liberación del mal, del pecado y de la muerte; por él, Dios da la "vida nueva", divina y eterna. Esta es la "Buena Nueva" que cambia al hombre y la historia de la humanidad, y que todos los pueblos tienen derecho a conocer (RM44). 


\section{Universidad, iglesia y cultura}

La incidencia académica, la pastoral de la Iglesia constituyen aspectos fundamentales para ahondar en la dimensión de evangelizar la cultura, que, en sí, es dejar que el Evangelio impregne la vida concreta de los hombres y mujeres de una sociedad dada. "Para ello, la pastoral ha de asumir la tarea de imprimir una mentalidad cristiana a la vida ordinaria" (Ecclesia in Europa, n. 58). Al referirnos a la cultura, en el sentido etimológico proviene de cultivo, "consiste en la creación de nuevas formas de cultivar las relaciones entre los seres humanos mismos y la naturaleza para incrementar las posibilidades humanas de vivir en paz" (Enciclopedia de paz y conflictos. Tomo I. 2004, pp. 208-209). Asimismo, la Iglesia en la Evangelii Nuntiandi, resalta algunos ámbitos, que:

...dispone de una imponente red de centros de enseñanza, desde la escuela elemental a la Universidad. A diario, las escuelas y centros de enseñanza católicos congregan a millones de jóvenes. Este hecho constituye una excelente oportunidad, a condición de que se la aproveche para proponer una formación auténticamente cristiana, donde la fe se convierte en el elemento unificador de todas las actividades del Instituto. En numerosos países, la enseñanza de la religión católica en las escuelas públicas está garantizada, con cotas que alcanzan a veces hasta el 90\% del total de alumnos, como es el caso de Italia... La ignorancia, ya sea religiosa o cultural, es una de las causas principales del increencia, de la mal- creencia y de la indiferencia religiosa. Para hacer frente a la ignorancia, es necesario replantearse las diferentes formas de educación y de formación actuales, especialmente en el nivel elemental. El papel de los maestros, que tienen que ser además testigos, es esencial. Siempre es buen momento para enseñar, como lo muestran los Evangelios, que presentan a Jesús dedicado a hacerlo durante la mayor parte de su vida pública... En este campo, es importante definir mejor la especificidad cristiana frente a la Nueva Era, a las sectas y a los nuevos movimientos religiosos, tanto en el nivel de la investigación teológica como en el de la formación de los catequistas. La superstición y la fascinación por la magia son a menudo resultado de una formación insuficiente. La ignorancia de los contenidos esenciales de la fe favorece el crecimiento de las sectas y la multiplicación de los falsos 
profetas. La presencia de la Iglesia en las Universidades, tanto en el campo de la enseñanza como en el de la pastoral, es vital. Aun cuando no esté presente a través de una Facultad de Teología, la Iglesia asegura su presencia a través de una pastoral universitaria, que se distingue de la simple pastoral juvenil. La pastoral universitaria apunta principalmente a la evangelización de la inteligencia, la creación de nuevas síntesis entre la fe y la cultura y se dirige prioritariamente a los profesores y docentes, para disponer de católicos bien formados (EN, 21-24).

Es fundamental indicar que lo propio la misión y visión de una universidad católica y de la Iglesia es el humanismo de Cristo que se desprende de la vivencia del evangelio. En este sentido, el mismo Papa Juan Pablo II en la Ex Corde Ecclesiae, subraya algunas características de la Universidad Católica, afirmando que constituye una:

Inspiración cristiana, no solo de parte de cada miembro, sino también de la Comunidad universitaria como tal; acentúa una reflexión continua a la luz de la fe católica, sobre el creciente tesoro del saber humano, al que trata de ofrecer una contribución con las propias investigaciones; la fidelidad al mensaje cristiano tal como es presentado por la Iglesia y el esfuerzo institucional al servicio del pueblo de Dios y de la familia humana en su itinerario hacia aquel objetivo trascendente que da sentido a la vida (ECE, 13).

Para el Cardenal Cardenal Zenón Grocholewski (2002), prefecto de la Congregación para la Educación Católica en su artículo la "Universidad como proyecto evangelizador", indica:

Es un hecho que se debe partir de la identidad de la Universidad católica que implica necesariamente, su aspecto evangelizador; que se resalta una serie de consideraciones sobre la identidad fundamentados en la Constitución Ex Corde Ecclesiae, los aportes del Santo Padre Benedicto XVI; todo lo anterior con el maravilloso fruto de la V Conferencia General del CELAM en Aparecida, Brasil. Del mismo modo, la evangelización de las personas; la evangelización de la cultura y la inculturación del evangelio, todo ello desde la perspectiva del ser y del quehacer propio de la universidad católica, y el llamado de Aparecida de convocar a las Universidades Católicas para que sean cada vez un lugar de irradiación del diálogo entre fe y razón. (ECE, 498). 
Según el enunciado anterior, el Cardinal Newman añade, en su Gramática del asentimiento:

La importancia del doble canal de la evangelización, el corazón y la cabeza, es decir, el sentimiento y la razón. Hoy día, la dimensión emocional de la persona adquiere importancia creciente y numerosos cristianos llegan por este medio al gozo de la fe. En una cultura de irracionalismo dominante, experimentan la necesidad de profundizar sus razones para creer mediante una formación apropiada, donde la Iglesia se hace "samaritana" de la razón herida (EN, 29).

En el mismo contexto anterior, el Papa Benedicto XVI en su Discurso dado en el Encuentro con los educadores católicos. (2008) respecto de la Universidad Católica de América realizado el 17 de abril del mismo año en Estados Unidos de América, enfatiza:

La realidad comunitaria de la universidad, su vocación a ser universitas, se encuentra en el eje de su identidad. En este sentido, al dirigirse a la comunidad de educadores católicos en su visita a los Estados Unidos el Santo Padre afirma que: La misma dinámica de identidad comunitaria - ¿a quién pertenezco? - vivifica el ethos de nuestras instituciones católicas. La identidad de una Universidad o de una Escuela católica no es simplemente una cuestión del número de los estudiantes católicos. Es una cuestión de convicción: ¿creemos realmente que solo en el misterio del Verbo encarnado se esclarece verdaderamente el misterio del hombre (cf. GS, 22)? ¿Estamos realmente dispuestos a confiar todo nuestro yo, inteligencia y voluntad, mente y corazón, a Dios? ¿Aceptamos la verdad que Cristo revela? En nuestras universidades y escuelas ies "tangible" la fe? ¿Se expresa fervorosamente en la liturgia, en los sacramentos, por medio de la oración, los actos de caridad, la solicitud por la justicia y el respeto por la creación de Dios? Solamente de este modo damos realmente testimonio sobre el sentido de quiénes somos y de lo que sostenemos.

La felicidad ontológica o mística es un don que viene cuando el ser humano es capaz de donarse, de practicar las virtudes. No podemos ir edificando un ser humano vacío sino una persona en proceso de permanente conversión, de ir reconociendo lo humano y lo trascendente. A esto se suma los aportes del Papa Francisco en 
sus escritos, sobre todo su preocupación de una reevangelización en Europa, el fortalecimiento en América Latina, el aporte que debe hacer la academia y la presencia de los laicos comprometidos en los diferentes ambientes pastorales.

\section{Ahondar en la realidad personal}

Es fundamental iluminar la problemática concreta y existencial primero de cada uno, porque "nadie da de lo que no tiene sino de lo que abunda en lo profundo del corazón”, de nuestro ser. De esta manera, podemos comprender, a nuestro entender, dentro de la más amplia filosofía moral, es decir, desde el ámbito de las actitudes y comportamientos de la persona, conforme lo indica Llano (2005), en su libro La vida lograda. ¿Quién no quiere mirar a su vida y poder decir algo semejante de ella? Todos aspiramos a vivir una vida completa, entera, lograda. Eso está muy claro. Y también lo está que no todos lo conseguimos. De hecho, hay personas felices y personas desdichadas. Algunas logran su vida y otras la malogran, la pierden, fracasan en ella. No fracasan en este o en aquel aspecto, sino que fracasan en sí mismas, precisamente como personas.

Panasiuk (2006, p. 15) enfatiza que "cada uno de nosotros debemos aprender a estar contentos y a disfrutar de la vida sin importar el lugar en el que estemos o la situación que se nos presente en la vida”. Aprender a ser felices significa dar lo mejor de nosotros mismos, poner en práctica nuestros talentos, dones que la vida nos ha dado, es decir, hay que trascender, servir, enriquecer la vida de nuestra esposa (o), amar y enseñar a nuestros hijos lo mejor y sobre todo desarrollar, potenciar y profundizar en la vida espiritual, unida a la práctica de valores y virtudes.

Bill (2009) dice que la mayoría de las personas se les dificulta expresar con claridad sus opiniones, deseos o necesidades a otra persona, al mismo tiempo cuando tienen momentos de felicidad o infelicidad que siente en un determinado momento de su vida. De acuerdo a nuestro vocabulario describir el término felicidad nos fal- 
taría tiempo para comprender el mismo. Sin embargo, algunos autores describen: Felicidad como (dicha, éxtasis, gozo, satisfacción, alegría entre otros); Infelicidad como miseria, desesperación, intranquilidad, tristeza (p. 10).

En esta misma orientación Rivadeneira (2014) indica:

Que si tenemos metas para nuestra vida, qué importante es cumplir las mismas, pero, partiendo de nuestras propias capacidades. Tu proyecto de vida es importante, porque vas a señalar lo que quieres ser en el futuro. En la vida, es importante tener visión de futuro; es decir, quienes logran esta visión se superan en la vida (p. 1).

En tal virtud esto implica hacer un recorrido honesto, generoso, exigente, crítico y valorativo por lo que ha sido la propia vida, buscando identificar las características personales, cuáles de ellas pueden considerarse como fortalezas y cuáles como debilidades. De esta manera, es importante incidir en una concepción más humana e integral de la persona desde la propia familia, es decir, no se puede concebir al ser humano solamente desde su corporalidad o únicamente desde su espiritualidad, dado que la persona, es una realidad única e integral conformada por una dimensión psicosomática (física, psíquica y espiritual); y por ello, la importancia y sentido por su propia existencia, por su origen, su desarrollo, su finalidad y su proyección trascendente, como permanente búsqueda de superación y perfección.

\section{A modo de conclusiones}

Una vez realizado un análisis y reflexión respecto del anuncio del kerigma, la incidencia de la iglesia, universidad, cultura frente a la increencia e indiferencia religiosa, a modo de reflexiones finales, es prioritario:

- El fortalecimiento de la formación ontológica, es decir del ser, en todas sus dimensiones que le permita discernir su vida frente a una cultura del tener, hedonista, relativista, 
reduccionista, que muestra a un ser humano que se degrada, asimismo.

- Es tarea de universidad y la iglesia, en el ámbito de la ciencia, la investigación, la axiología, profundizar, vivenciar el sentido de la experiencia del encuentro con Cristo en la propia vida y del prójimo, el más cercano, el que vive en situaciones vulnerables, es decir, hay que detenernos a conocer mejor la realidad en la que vivimos y hacer urgente algo que sea visible y luego saber hacia dónde vamos, para contribuir a un mundo real diferente, sin prejuicios, más humano y digno.

- La Iglesia en sus diversos documentos como: Encíclicas, Exhortaciones, Cartas, el Catecismo de la Iglesia Católica y la misma doctrina, deja claro los principios del evangelio frente a las manifestaciones religiosas y culturales, que sin duda está expuesta a ciertas carencias, limitaciones, interpretaciones distorsionadas de las creencias, de la religión, como es el caso de las supersticiones, la religiosidad popular, sacramentos. En este mismo contexto sino hay una depuración y formación pertinente desde la Iglesia y la universidad, únicamente puede quedarse a un nivel de manifestaciones culturales, sin llegar a una adhesión de la fe.

- Las experiencias religiosas en la vida del creyente bien formadas contribuyen al fortalecimiento de la creencia, la fe, a pesar que ciertamente hay ciertos hechos que muestran la falta de coherencia entre el creer, pensar y vivenciar el evangelio en un sentido más serio de compromiso personal y comunitario. Sin embargo, cuando la creencia está vinculada activamente con la práctica, la misma está bien orientada, comporta un hondo sentido de los atributos profundos de Dios, engendra actitudes interiores, refleja una sed de Dios, hace capaz de generosidad y conversión de vida, lo cual cada vez engendra un verdadero encuentro con Dios en Cristo y en la vida cristiana. 


\section{Recomendaciones}

Se debe mejorar en algunos aspectos en cuanto a la religiosidad, la participación de los sacramentos o vivencias religiosas de los fieles, aunque su intención a lo mejor es buena, sin embargo, hay cosas que se observan que no guardan coherencia con los principios cristianos, y que en algunos casos ha llevado más a la indiferencia, increencia y a un relativismo religioso.

La iglesia debe profundizar y proporcionar una formación permanente, con la finalidad de depurar, cambiar ciertas actitudes y comportamientos de los fieles, permitiendo de esta manera una interiorización de la fe en relación con la liturgia, la doctrina, la vivencia de los sacramentos, y sobre todo llevar a los fieles a una profunda vida espiritual que se identifique sinceramente con el evangelio, en una fe encarnada en el diario vivir.

Estructurar un proyecto integral de formación pastoral desde la Iglesia a nivel de cada diócesis, en el caso de la universidad, una pastoral de servicio, de vinculación con la colectividad en situaciones vulnerables (participación de docentes, administrativos y estudiantes).

Promover desde la universidad junto a la iglesia investigaciones, formación específica según las necesidades sociales, círculos de estudio en ámbitos de doctrina, liturgia, biblia; difusión, socialización de proyectos sociales, utilización de los medios de comunicación para programas de radio, televisión y publicación de artículos en revistas de divulgación y científicas.

\section{Bibliografía}

Bill, L. (2009). ¿¡Familias felices!? SOS padres en apuros. Claves para conseguir un entorno feliz. Traducido al español por Francisco Domínguez Montero. España: Editorial EDEBE.

Catecismo de la Iglesia Católica (1993). Ciudad del Vaticano: Librería Editrice Vaticana.

Consejo Episcopal Latinoamericano, CELAM (2012). Evangelización y cultura de América Latina. 
Consejo Pontificio para la Cultura (2004). Documento final de la última asamblea plenaria del Consejo Pontificio para la Cultura. (11-13 de marzo).

Concilio Vaticano II. Constitución Dogmática "Sacrosantum Concilium". Constitución pastoral "Gaudium et Spes".

De Angelis, B., y Zordan, P. (2006). Antropología. Loja: Editorial UTPL.

Discurso de Su Santidad Benedicto XVI (2008). Encuentro con los educadores católicos. Universidad Católica de América. 17 de abril, Estados Unidos.

Gevaert, J. (1984). El problema del hombre. Salamanca: Ediciones Sígueme.

Girardi, J. (1967). Reflexiones sobre la indiferencia religiosa. Concilium, 23, 439-449. LIÉGÉ P.A

González de Cardedal, O. (1985). La gloria del hombre. Reto entre una cultura de la fe y una cultura de la increencia. Biblioteca Autores Cristianos. EncíclicaRedemptorisMissio(esp.Cap.I.nn,4,9,12,13,32,35,36,42,47,52,59). Iglesia y liberación humana (1971). Los Documentos de Medellín; II Conferencia General del Episcopado Latinoamericano. Edición autorizada por el CELAM. Quito: Editorial Don Bosco.

INEC (2012). Primeras estadísticas sobre filiación religiosa en el Ecuador. Agosto 2012. Quito: Instituto Nacional de Estadísticas y Censos. Disponible en: www.inec.gob.ec

Izquierdo, C. (2006). Increencia. En: Diccionario de teología. Pamplona: Eunsa. Juan Pablo II (1990). Ex Corde Ecclesiae, sobre las Universidades Católicas, Juan Pablo II, Constitución Apostólica (15 de agosto de 1990). (1998). Carta encíclica Fides et Ratio sobre las relaciones entre Fe y Razón. Roma-Italia.

(2002). Directorio sobre la Piedad Popular y la Liturgia. Principios y Orientaciones. Ciudad del Vaticano.

Llano, A. (2002). La vida lograda. España: Editorial Ariel, S.A.

Pablo VI. (1975). Exhortación Apostólica "Evangelii Nuntiandi” (8 de diciembre de 1975).

Panasiuk, A. (2006). ¿Cómo llego a fin de mes? Grupo Nelson (una división de Thomas Nelson Publishers). Nashville, Tennessee, Estados Unidos de América.

Rivadeneira, L. (2014). ¿Cómo hacer un proyecto de vida? EcuadorUniversitario.com. Quito.

Sánchez Hernández, O. (2008). La pluralidad como principio. Anotaciones para una Teología Fundamental Pluralista. En: Cuestiones de 
Teología en el inicio del siglo XXI (pp.101-131). Colombia: Edición Universidad de San Buenaventura.

Secretariado de la Comisión Episcopal de Enseñanza. (2001). Educar desde el Evangelio. Sentido evangelizador y eclesial de la enseñanza religiosa escolar y sus profesores. Madrid: Editorial EDICE.

Tamayo, J. J. (2004). Fundamentalismos y diálogo entre religiones. Madrid: Trotta.

UNESCO (1997). Nuestra diversidad creativa. Informe de la Comisión Mundial de Cultura y Desarrollo. Madrid: Fundación Santa María/SM, p. 9

Vitoria, J. (2012). Vientos de cambio. La Iglesia ante los signos de los tiempos. Colección Cuadernos 137. Cristianismo y Justicia. Barcelona: Ediciones Rondas.

\section{Siglas de documentos eclesiales}
AA Apostolicam actuositatem
AG Ad Gentes
LG Lumen Gentium
CL Chrfistifidelis Laici
CT Catequesi Tradendae
RM Redemptoris missio 